\title{
Was könnte Grundtvig heutzutage für reformierte Christen bedeuten?
}

\author{
Uber Grundtvig in Holland \\ von G.F.W. Herngreen
}

"Grundtvigstudiers“ Redaktion hat mich gebeten, zu versuchen, diese Frage mit einer ersten, vorläufigen Antwort zu versehen. Mehr ist meines Erachtens nicht möglich. Grundtvig war in den Niederlanden bis vor kurzem in Kirche und Theologie fast völlig unbekannt. Nur in Volkshochschul-Kreisen wusste man von ihm und seinen Gedanken über Erziehung der reiferen Jugend, ohne jedoch viel Verständnis zu haben für den ganzen Grundtvig, und für die Zusammenhänge zwischen seinen erzieherischen, völklichen und vor allem kirchlich-theologischen Gedanken.

Diese Unbekanntheit beruhte bei uns zuerst einmal einfach auf sprachlichen Gründen. Nur wenige Theologen in Holland sind der dänischen Sprache Meister. Skandinavische Theologie hat uns im allgemeinen via deutsche Übersetzungen erreicht. So wurde Kierkegaard hier bekannt; auch heute noch wird er oft auf Deutsch zitiert obwohl es gute holländische Übersetzungen aus dem Dänischen gibt; z. B. eine vorzügliche, annotierte Ausgabe der „Filosofiske Smuler". Bei der Lektüre Kierkegaards fand man dann auch Grundtvig genannt; aber das vernichtende Urteil das K. über ihn spricht - ohne ihm wirklich gerecht zu werden und ohne sein tiefstes Anliegen verstanden zu haben -, hatte zur Folge, dass Grundtvig für die vielen die K. lasen, schon erledigt war bevor er zu Worte hatte kommen können.

Die niederländische Theologie hat Grundtvig völlig übergangen. In keiner Dogmatik wird er auch nur genannt. Seine "mageløse Opdagelse" ist keinem holländischen Schriftgelehrten je zur Anfechtung geworden. Handbücher der Kirchengeschichte nennen ihn schon - der holländische Prof. Bakhuizen van den Brink gibt in einigen Zeilen ein nicht unbefriedigendes Bild - aber noch immer 
wird das deutsche „Kompendium der Kirchengeschichte“ von Heussi sehr viel benutzt. In diesem Kompendium fand sich vor dem letzten Weltkrieg die groteske These, Grundtvigs theologische Einsichten (von 1825!, Rekurs auf das Apostolikum) wären seinem „Deutschenhass" nach dem Konflikt 1848/49 zu verdanken ...! Nach dem Kriege kam dieser Nonsens in Wegfall, aber noch immer zeugt „Heussi's“ Tekst von totalem Unverständnis.

Sehr viel Einfluss hatte Karl Barth. Leider hat auch er Grundtvig vernachlässigt, indem er ihn nur ein einziges Mal nennt, und dann noch nur „als den im 19ten Jahrhundert deutlichsten wirklichen Vorläufer der modernen Volks- und Schöpfungsordnungentheologie." Anscheinend hat Barth nichts von Grundtvig selbst gelesen: seine Information bezieht er aus zweiter Hand: auf die deutsche Übersetzung von Hans L. Martensens "Soziale Ethik", 1888 ... Dazu hat Barth auch die deutsche Ausgabe von N. H. Søe's „Kristelig Etik“ gebraucht. Barth schrieb seine Zeilen über Grundtvig in 1951 (Kirchliche Dogmatik III/4, S. 346/347) als er schon 15 Jahre lang die Gelegenheit gehabt hatte, den Artikel über „Die Frage nach einer theologischen Grundtviginterpretation" zu lesen, den Regin Prenter, damals noch Pastor schon in 1936 in dem Barth zum 50sten Geburtstag gewidmeten Sammelband publiziert hatte. Hätte Barth das getan, so hätte er sich sicher davor gehütet mit einer Grundtvig-Konstruktion zu arbeiten die auf typisch deutschen - und von Barth mit Recht bestrittenen - Voraussetzungen beruhte, die Grundtvig aber völlig fremd waren.

Es wäre bestimmt interessant und fruchtbar gewesen, wenn Barth die wirklichen Gedanken Grundtvigs, zumal seine kirchlichtheologischen, gekannt und sich damit auseinandergesetzt hätte. Dabei denke ich vor allem an beider Lehre vom Worte Gottes, dem lebendigen schöpferischen Herrenwort das, bei allen Unterschieden die es hier sicher gibt, sowohl bei Grundtvig als bei Barth nicht die H. Schrift sondern zuerst der lebendige Herr Selber ist. Hier lagen und liegen immer noch oekumenische Möglichkeiten. Weiter sei hier nur noch Barths Lehre von der guten Schöpfung Gottes genannt.

Wenn man in Holland beim Nennen des Namens "Grundtvig“ sich überhaupt etwas vorstellen konnte, dann war es das Bild eines romantischen, patriotischen, national-christlichen, volkskirchlichen 
Dänen; und wo auch Holland die enge Verbindung von Nationalem und Christlichem nur allzu gut kannte, und die kritische Theologie sich ernsthaft genötigt sah, die selbstsicheren Verbindungen und Identifikationen die es hier bis in die Politik hinein gab, zu lockern, brauchte man einen solchen Grundtvig bestimmt nicht. Im besten Fall sah man ihn als eine Réveil-figur, die gegen den Rationalismus gekämpft hatte, aber solche Figuren hat es auch in Holland in Hülle und Fülle gegeben!

Weiter: Wie karakteristisch Grundtvigs Gedanken auch sein mögen, im grossen und ganzen steht er in der lutherischen Tradition, und seine Stellungnahmen sind vielleicht zum Teil nur von lutherischen Voraussetzungen aus völlig verständlich $\mathrm{zu}$ machen. Auf jeden Fall ist es unentbehrlich, einige tief verwurzelten Missverständnisse auf beiden Seiten wegzuräumen, bevor es zu einer wirklichen Begegnung kommen kann. Meiner Meinung nach bietet gerade Grundtvig hier, trotz seiner scharfen Abwehr eines übrigens teilweise falsch verstandenen Calvinismus, originelle Möglichkeiten!

Schliesslich: das starke dänisch-nationale Selbstbewusstsein das längere Zeit zumal grundtvigsche Kreise prägte, zeigte sich als ich, damals noch Student, in 1936 eine Woche in Askov verbrachte, und die Meinung äusserte, Grundtvig könne auch ausserhalb Dänemarks, z. B. in Holland, fruchtbar gemacht werden. Man versicherte mir: „Grundtvigianismus sei kein Exportartikel“. Wäre das richtig, so hätten Grundtvigs Gedanken ohne Weiteres ihren Wert verloren: eine Lehre, die sich als wesentlich christlich gibt, kann gerade deswegen nicht auf ein Volk beschränkt bleiben; wohl kann sie eine starke nationale Färbung haben, und auch inhaltlich bestimmte Ideen über Bedeutung und Wert des Volkes umfassen, aber in ihrem tiefsten Wesen muss etwas Essentiëlles vorhanden sein das alle nationale Schranken transzendiert - sonst wäre sie keine christliche Lehre sondern eine nationalistische Haeresie. Ich habe mich darum damals, des besseren belehrt (u. a. durch A. Nørgaards Publikationen), in Askov nicht abschrecken lassen, und immer geglaubt, Grundtvig habe der gesammten Christenheit, einschliesslich der Reformierten, in allen Ländern Wesentliches zu sagen. Seine Gedanken sind keine national-dänischen Besonderheiten, sondern christliche Akzente, die in der ganzen oekumenischen Diskussion mit aufgenommen werden sollen. Und dabei sollte gerade 
das meist Charakteristische, in dem Grundtvigs Glaubensbekenntnis zu Lobgesang wird, seine überreiche Liederdichtung, auch für die Kirchen ausserhalb Dänemarks fruchtbar gemacht werden!

Dass das möglich ist, (trotz den in "Grundtvigstudier 1975“ Seite 68 von Uffe Hansen genannten Schwierigkeiten), ist in Holland deutlich geworden. Grundtvig hat, gerade in der Gestalt des grossen "Psalmist" nun doch endlich seinen Eintritt ins kirchliche Leben in den Niederlanden gemacht! Fünf Lieder wurden schon am Anfang der 1960er Jahre von Muus Jacobse (Dichtername des leider zu früh verstorbenen Professoren K. Heeroma) in holländische Sprache übersetzt mit Beibehaltung des ursprünglichen Rhytmus und der dänischen Melodie. Drei dieser fünf wurden von Heeroma, der Mitarbeiter am neuen holländischen „Liedboek" (Gesangbuch) war, der Redaktion zur Aufnahme angeboten. Es waren: „Herren Han har besøgt sit Folk“; „Vor Herre, til Dig må jeg ty“; und eine besonders gute Übersetzung von „Kirken den er et gammelt Hus", die aber unbegreiflicherweise nicht aufgenommen wurde, obwohl es in fast alle modernen Sprachen übersetzt ist und eine gewisse oecumenische Bedeutung bekommen hat. Und weiter bestehen Pläne, in den kommenden Jahren mehrere Grundtviglieder zu übersetzen.

Die Aufnahme zweier Grundtviglieder im neuen, in 1973 erschienenen „Liedboek der Kerken“ - es wird von den fünf wichtigsten Kirchen in Holland benutzt: Die Nied.Reformierte Kirche, die (freien) Reformierten Kirchen in den Niederlanden, die Evang. Lutherische Kirche, Die Taufgesinnte Sozietät (Mennoniten), und die Remonstrantisch Reformierte Kirche (entstanden in 1618/19, als Protest gegen die damals so harte Praedestinationslehre) - veranlasste zu einigen Artikel über die theologischen Hintergründe dieser Lieder im reformierten Amtsblatt. In 1975 wurden auch vier kurze Radiovorträge über Grundtvig gehalten, die später gedruckt wurden. (G.F.W. Herngreen, „De betekenis van de deense dichter-theoloog N.F.S. Grundtvig" in "Rondom het Woord“ 1975, nr. 4). Das „Heutzutage“ im Titel dieses Beitrages bekommt so einen Sinn: Es gibt eine, sei es noch sehr schmale, Basis für eine Grundtvigdiskussion im reformierten Holland.

Das erste Thema, das von seinen beiden Liedern her zu erörtern 
war, betraf Grundtvigs Lehre vom lebendigen Worte Gottes in Verhältnis zur Heiligen Schrift. Was Grundtvig, in seiner Opposition gegen das übliche protestantische Schriftprinzip, zu sagen hatte, trifft den ganzen Protestantismus, Lutheraner und Reformierte zusammen, Rationalisten wie in 1825 und eine neu vorrückende Bibelorthodoxie, wie in den Jahren nach 1860. Das herkömmliche Schriftprinzip, mit seinen „toten Buchstaben, die - Kernpunkt der Theologie Grundtvigs - nichts vermögen“, ruft unzählige Fragen der Authentizität der Auslegung und der Anwendung hervor; es liefert uns einem papierenen Papste aus, der, wie der Papst zu Rom, von vielen Prälaten umgeben ist: es sind die Schriftgelehrten, Theologen, Pfarrer, von denen ein schlichtes Mitglied der Gemeinde dann weiter für Seele und Seligkeit in Leben und Sterben abhängig wird, es sei denn, wie wir heute mehr und mehr erleben, dass es sich selbst zu Prälaten macht oder sogar sein eigener Papst wird.

Grundtvig hat recht verstanden was Karl Barth später so formulierte: dass Orthodoxie und Rationalismus „Kinder desselben schwachen Geistes" sind. Das Wort ist Fleisch geworden, nicht Schrift. Die Kirche ist nicht auf der Schrift gebaut, die Linie führt nicht von Gott und Christus via die Schrift zur Kirche oder vielleicht sogar geradeaus zum Einzelnen. Die Gemeinde war da, vom ersten Anfang an, mit dem Herrn selbst in ihrer Mitte, „licht und lebendig anwesend, „lyslevende til stede““-. Dort wurde nicht über Ihn gesprochen, dort redete Er Selber in Worten, die Geist und Leben sind, bei Taufe und Abendmahl, lange bevor auch nur ein Buchstabe des Neuen Testamentes geschrieben war. Die Kirche beruht nicht auf der Schrift, sondern die H. Schrift wächst in der Kirche; sie hat ihre "Traditionsgeschichte“, würden wir heute sagen; sie ist das Zeugnis hinsichtlich des Glaubens und der Lehre der lebendigen Kirche, ihr Unterrichtsbuch.

Es gilt, zumal in reformierten Kreisen, deutlich zu machen, dass diese "historisch-kirchliche Sicht" Grundtvigs der Bibel ihren Wert auf keinen Fall wegnimmt. Sie ist einzigartig. Aber den Glauben schenkt sie nicht: das tut allein der lebendige Herr Selber durch seinen Geist im lebendigen Wort das in der Gemeinde tönt. Der Herr schreibt uns nicht an, er redet uns an. Immer wieder zeigt Grundtvig auf die vielen Bibeltekste die ein lebendiges, mündliches Herrenwort in der Gemeinde voraussetzen. Die Bibel interpretiert 
es, ruft uns auf, aus diesem Herrenwort zu leben, aber sie IST nicht dieses Wort selbst. Ob Grundtvigs allgemeine Sicht auf das Verhältnis zwischen geschriebenem und gesprochenem Wort hier eine primäre Rolle spielt, kann hier unentschieden bleiben. Sein Umgang mit Mythus und Sage hat ihn auf jeden Fall in der Überzeugung gestärkt, dass Worte keine Formeln für Begriffe sind; Sprache ist keine Semantik sondern Poëtik: sie ruft Wirklichkeiten auf, und spricht von Herz zu Herz. Der dichtende, singende „Skjald“ steht ihm vor Augen, in lebendiger Wechselwirkung mit seiner Zuhörerschaft.

Der tiefste Grund aber für Grundtvigs Suchen nach einem direkten Worte des Herrn Selbst liegt meines Erachtens in der Nähe von Luthers Frage: „Wie bekomme ich einen gnädigen Gott?" Jeder der in der Schrift auf der Suche ist nach persönlichem Angeredet-werden, kann leicht in Verwirrung geraten: Was ist für mich gemeint? Und was meint es? Damit fällt er wieder in die Hände der Prälaten des papierenen Papstes. Aber das lebendige "Mundwort" des Herrn Selbst begegnete Grundtvig in den lapidären Sätzen der Liturgie der Taufe und des Abendmahls: „Glaubst Du ...? Ich taufe Dich .. Vater Unser ... Nehmet, esset, trinket ... Friede sei mit Euch!“ Grundtvig erlebt es wie eine überwältigende Befreiung, die den Ton seiner Lieder weithin beherrscht. Für ihn handelte es sich um den Guten Hirten Selbst, der seine Schafe mit Namen ruft, jedes mit seinem eigenen, persönlich. Daher die vielleicht überspitzten und oft bestrittenen Worte: „Kun ved Badet og ved Bordet hører vi Guds Ord til os“ - Nur bei Bad (Taufe) und Tisch (Abendmahl) hören wir Gotteswort $z u$ uns. - zu úns, persönlich, das „Wort, das schafft, was es nennt" und nicht zuerst Schrift werden sollte, sondern von Anfang an durch die Jahrhunderte hindurch in der Gemeinde lebendig gesprochen übertragen wird. Man hat diesen existentiëllen Karakter der grundtvigschen These oft übersehen. Und dennoch klingt gerade er uns aus all seinen Liedern unüberhörbar entgegen.

Diese Konzentration auf die sakramentalen Worte als eigentliches Herrenwort, einschliesslich des Apostolikums und Vater Unsers - wir kommen darauf noch zu sprechen - ist für die reformierte Orthodoxie problematisch, aber nicht problematischer als für die lutherische. Es gibt sogar auch im reformierten Protestan- 
tismus Anknüpfungspunkte, die ein Verständnis für das tiefste Anliegen Grundtvigs ermöglichen. Für Lutheraner und zumal für Grundtvigianer die dazu noch in „Grundtvigstudier 1975“ Grundtvigs scharfe Abwertung der reformierten Abendmahlslehre gelesen haben (Seite 15) möchte das verwunderlich scheinen. Aber gerade hier herrschen hartnäckige Missverständnisse hinsichtlich der reformierten Abendmahlslehre, die endlich einmal aufzuräumen sind.

Grundtvig war, wie so viele Lutheraner, der Meinung, die Reformierten machten mit ihrem "significat" (Brot deutet auf den Leib Christi, weist nur hin, ist "nur" Zeichen) statt „est“ (Brot wird Leib, oder „in, mit und unter" Brot ist der Leib Christi) eine Aussage, in der sie die wirkliche Gegenwart Christi im Sakrament leugneten, und das ganze Abendmahlsgeschehen reduzierten auf eine Erinnerungsfeier ohne Präsenz, in der nur der Glaube sich auf Vergangenes richtet und sich daran emporzieht ohne dass von Gottes Seite her etwas Wirkliches geschieht.

Es ist an der Zeit, dieses Missverständnis für immer zu beseitigen. Bei allen Unterschieden die es zwischen lutherischer und calvinischer Abendmahlslehre gibt, auch die calvinische Lehre weiss, dass es im Sakrament um ein Handeln Gottes geht, um wirkliche heilsame Gegenwart Jesu Christi Selbst.

Auch Grundtvig bringt, wenn er über die Elemente Brot und Wein redet, das "significat" in Anwendung (Siehe wieder den Beitrag Chr. Thodbergs in Gr. St. 1975). Die leibliche, die vollpersönliche Gegenwart Christi findet er im Abendmahlswort; das ist die Anrede des Guten Hirten an Seine Herde und in dieser an jedes Mitglied persönlich, in der Er "lyslevende“ gegenwärtig ist. Wir können es vielleicht so sagen: das Materielle, das Nehmen, Fühlen, Essen, Schmecken in seiner ganzen Sinnlichkeit, dieses leibliche Geschehen besiegelt die Wahrhaftigkeit dieser persönlichen Präsenz, und ist ebenso unentbehrlich wie die Umarmung des Vaters und sein Kuss im Gleichnis vom verlornen Sohn (Luk. 15) neben den entscheidenden WORTEN: „Dieser mein Sohn!“ Nur Worte ergäben einen distanzierten Platonismus; gerade die Umarmung und der Kuss sind der Ausdruck einer totalen, den verlornen Sohn in seiner ganzen konkreten Existenz umfassenden vergebenden Liebe.

Die Calvinisten haben mit ihrem „significat“, wie Grundtvig, 
nie die wirksame Gegenwart des ganzen Christus im Abendmahl leugnen wollen. Sie fühlen sich von Grundtvigs Kritik in dieser Hinsicht darum auch nicht betroffen.

Der Akzent-Unterschied im allgemeinen zwischen lutherischer und calvinischer Lehre liegt vielmehr darin, dass bei Luther die Frage der Heilsgewissheit zentral war, während für Calvin die Souveränität Gottes im Mittelpunkt stand. Deshalb hütet richtige calvinische Theologie sich immer vor jeder Aussage die den Eindruck machen könnte, wir Menschen könnten über Gott, über Seine Gegenwart verfügen - wie z. B. in der römischen Messe durch Consecration und nachfolgende Transsubstantiation-. Wenn der Herr mit uns, bei uns ist, so ist er das in Seiner eigenen souveränen Freiheit, wann, wo und wie es Ihm gefällt. Das liess sich am besten sagen mit den Worten: „Per Spiritum sanctum“ - durch den Heiligen Geist-, als Modus der Gegenwart Christi. "Geist" bedeutet: souveräne, freie Tätigkeit Gottes, und ist unlöslich mit Gottes Wort verbunden. "Geist" meint also keine Spiritualisierung, Vergeistigung, Verdunstung der concreten Wirklichkeit, Entwirklichung, oder gar völlige Subjectivierung. „Per Spiritum Sanctum" heisst: Kraft Seines freien souveränen Wirkens ist der ganze Christus, der fleischgewordene, gekreuzigte und auferstandene Selber präsent, voll-persönlich. Man sehe hier auch die ausgezeichnete Ubersicht, die Yngve Brilioth in seinem „Nattvarden i evangeliskt Gudstjänstliv" unter 5. von "Den reformerta linjen“ gibt, speziell unter III: Den calvinska nattvardsuppfattningen, S. 233 - 241 und unter V. über De anglosaxiska samfunden, S. 263275 - die Schottische Theologie.

Dieser Nachdruck auf die souveräne Freiheit der göttlichen Tätigkeit beraubt uns nun aber, anders als man vielleicht denken möchte, nicht der Heilsgewissheit, die Luther zu seiner leidenschaftlichen Handhabung des "EST" in seiner Abendmahlslehre führte, und die auch Grundtvig, obwohl er die Akzente (auf gut calvinischer Weise!) von den an sich unentbehrlichen Elementen verlegte auf die Abendmahlsworte, so wichtig war. Calvins Lehre nimmt uns wohl jede Möglichkeit, die Gegenwart Christi von unserer Seite her zu garantieren - Gott ist der Freie, - aber Gottes Freiheit ist nie Willkür, sie hebt Seine Treue nicht auf: Er hält was Er uns in Treue versprochen hat. Darum weiss auch der Calvinist von der Heilsgewissheit, gerade im Sakrament ge- 
geben, dass „nicht nur andern sondern auch mir" Vergebung und ewiges Leben beschert sei. Auch im Calvinismus ist die ganz persönliche Stimme des Guten Hirten im Sakrament zu hören.

Für eine ausführlichere Analyse dieser Gedanken möchte ich den meines Erachtens besten, schönsten, tiefsinnigsten und mit tiefster innerlicher Anteilnahme und Liebe von reformierter Seite geschriebenen Aufsatz über "Ansatz und Absicht in Luthers Abendmahlslehre“ nennen. Er steht in „Die Theologie und die Kirche“, Gesammelte Vorträge Band 2, 1928; der Autor ist Karl Barth.

Fassen wir zusammen: Die reformierte Abendmahlslehre bietet bedeutsame Möglichkeiten für ein Verständnis des tiefsten Anliegens Grundtvigs: Wo hören wir Gottes Wort zu úns, persönlich. Auch Calvin hätte jeden Sonntag das Abendmahl gefeiert, wäre ihm der Rat der Stadt Geneve nicht mit einem Verbot entgegengetreten. Und heutzutage gibt es in der Reformierten Kirche eine zunehmende Anzahl Gemeinden, die das Abendmahl viel öfter als einmal in den traditionellen drei Montaten feiern. Dazu kommt, dass auch hier die von Chr. Thodberg in seinem erwähnten Artikel genannte Verengung auf eine fast exklusive Karfreitagsstimmung mehr und mehr überwunden wird und die von Thodberg genannten Momente: Gemeinschaft, vertikal únd horizontal!, Lobgesang und eschatologische Perspektive, zur Geltung gebracht werden.

Wir kehren zur Hauptlinie unseres Aufsatzes zurück. Die Verkennung des tiefsten "lutherischen" aber damit noch nicht uncalvinischen Anliegens Grundtvigs ist sehr deutlich in der - soweit man überhaupt etwas von ihm weiss - bei uns durchgängigen Beurteilung des Gebrauchs, den Grundtvig von Apostolikum macht. Allerdings ist er selber daran nicht ganz schuldlos. In den Spuren Irenaeus' entdeckt er es als „Richtschnur der Wahrheit". In „Kirkens Gjenmaele" und auch später fungiert es als Norm für die Beantwortung der Frage, wer zur Kirche gehören darf. Clausen sollte - jedenfalls damals noch - heraus! Dieser Gebrauch des Apostolikums stiftete Verwirrung, zumal als einer der Anhänger Grundtvigs die alte Legende einer mündlichen Überlieferung des Apostolikums von Jesus Selbst an die Apostel wieder ausgrub. Hier ist die Stelle, wo Kierkegaard Grundtvig, der dem Apostoli- 
kum eine so zentrale Position als Herrenwort gab, angreifen konnte. Auch das historische Faktum eines vom Herren Selbst stammenden Apostolikums gibt nicht den alle Unsicherheit ausschliessenden festen Punkt. Glaube ist nie „objektiv“ sondern immer ein Sprung, und alle historische Gewissheit ist immer approximativ, immer Gewissheit bis auf weiteres! Aber Kierkegaard hat nicht verstanden dass diese Theorie über das Apostolikum eine secundäre Rationalisierung einer primären Erfahrung, eines Erlebnisses war: eines direkt Sich-angesprochen-wissens.

Das ergeht aus einer merkwürdigen und vielleicht in diesem $\mathrm{Zu}$ sammenhang nicht genügend beachteten Aussage, die Grundtvig selber gemacht hat - aber was mute ich mir als nicht-Dänen zu ... vielleicht ist die Stelle schon oft zitiert? mir fiel sie jedenfalls als wichtig auf-. Sie steht in "Den christelige Børnelaerdom" im Kapitel „Det evige Livs-Ord af Vorherres egen Mund til Menigheden", Vaerker i Udvalg von Christensen und Koch Band VI Seite 239. Dort sagt er: „Es ist mir in einem ganzen Menschenleben nicht gelungen, sogar Professoren klar zu machen dass, wenn ich auf das Glaubensbekenntnis verweise, wie es seit vordenklichen Zeiten mit der Taufe verbunden war, ich das mit lauter Stimme gesprochene Ja und Amen der Gemeinde meine, und nicht einige alte Texte eines sogenannten Symbolum Apostolicum ...“ Das bedeutet: Es geht Grundtvig nicht um einen alten normativen Tekst auf Papier, sondern um einen gesprochenen Tekst, einen Tekst in aktivem Dienst! Das Apostolikum ist für Grundtvig kein klassischer Tekst, den man kommentieren kann, und den man als Statut einer Gesellschaft gebrauchen könnte; so wäre es ein totes Wort. Für Grundtvig ist das Apostolikum das lebendige Bekennen der Gemeinde von alters her, ja, aber héute erklingend, ein Bekennen in Actu; und so ist es zugleich das eigene, geschehende Wort des Herren, dort wo es erklingt, wo es ausgesprochen wird, wo es "geschieht". Das Apostolicum enthält keine Dogmata, sondern erzählt Wer Gott ist in dem was Er tat, tut und tun wird als Vater, Sohn und Geist.

Dass in Dänemark bei der Taufe das Apostolicum gerade nicht von der Gemeinde gesprochen wird sondern vom Pfarrer dem Täufling abgefragt wird, ist für Reformierte, die die Tauffragen immer an die Eltern stellen, schwierig zu verstehen. Vielleicht aber ist ihnen deutlich zu machen, dass gerade diese Form der 
Frage für Grundtvig wesentlich war: sie bedeutet, dass der Herr Selber ganz persönlich diesem neuen Mitglied seiner Gemeinde sagt, Wer Er für es sein will in dem was Er tat, tut, und tun wird als Vater Sohn und Geist, und es, bevor es seinerseits auch nur das Geringste Gott entgegenbringen kann - darum antworten vorläufig die Paten stellvertretend - liebevoll einlädt, Ihn anzunehmen wie Er ist. Keine Vorlesung konzentrierter Dogmatik also, kein Examen, in dem die Rechtgläubigkeit überprüft wird, sondern lebendiges Angebot einer Gabe die Er Selber ist.

Darum ist auch das übliche Urteil, Grundtvig habe das Apostolikum an die Stelle der Bibel gesetzt, völlig falsch. Er tat etwas ganz anderes. Er brach mit einer Anschauung des WORTES die, orthodox oder rationalistisch, das Wort als Mitteilung von Daten und Wahrheiten auffasste und setzte an deren Stelle das WORT als persönlichen, schöpferischen Aufruf. Dieses Wort, und in diesem den Herren Selbst fand er dann in der lebendigen, bekennenden Gemeinde, bei der Taufe im Glaubenswort (Apostolikum) und Taufwort, und dann im Vater-Unser, das ein Mensch, durch die Taufe Kind Gottes geworden, auf die Lippen gelegt bekommt als das Wort der Hoffnung; und dann kommt das Abendmahl mit dem Wort der Liebe - nehmet, esset, für euch - die die Grösste von allen ist, und schliesslich fasst der „Friede“ als Sein eigener Gruss alles zusammen. Sehr schön steht das beisammen in Grundtvigs schon erwähntem, leider nicht in das "Liedboek“ aufgenommenem Lied „Kirken den er et gammelt Hus“, mit seinem "Fred vaere med eder alle" am Schluss.

Aber bedeutet das nicht eine Rückkehr zum römischen Traditionsbegriff? Durchaus nicht. Wohl könnte man, mit Anders Nørgaard, sagen, dass Grundtvig einen Weg zurück suchte, hinter sowohl Katholizismus als Protestantismus. Aber Grundtvig kennt kein allmählich immer mehr anwachsendes "depositum fidei“, eben so wenig wie ein unfehlbares Lehramt. Die Lehre gehört zur Schule, und dort soll Freiheit herrschen. Möchte man dennoch von Tradition sprechen wollen, so würde es sich nur handeln um eine "Traditio pastoralis“, ein fortgehendes Weitertönen der Stimme des Guten Hirten in Seiner Gemeinde.

Grundtvigs Sicht hat, wie ich meine, noch immer ihre Bedeutung für das oekumenische Gespräch, auch mit Reformierten, die in den 
Niederlanden vielfach in Kontakt mit römisch-katholischen Gesprächspartnern sind. Grundtvig gibt dem Verhältnis von Schrift und Tradition einen speziellen Akzent. Er widersetzt sich einem sterilen Rationalismus sowie einer toten Buchstabenorthodoxie. Er sieht das Heilshandeln Gottes als redende Wirklichkeit. Er hat gebrochen mit allem hoch- oder staatskirchlichen Institutionalismus, verliert sich aber nicht im Sumpf einer Gemeindetheologie in der sich alles dreht um unsere fromme oder aktivistische Verbundenheit. Er verwirft den piëtistischen Individualismus, und nimmt als Ausgangspunkt seines Denkens die Gegenwart Christi in der Gemeinde als Ganzes, aber nicht ihrer eigenen frommen Erfahrung wegen: es handelt sich für ihn um die Gegenwart des Herren in den Worten der Taufe und des Abendmahls wie sie in der Gemeinde gefeiert werden. Das persönliche Erleben erhält innerhalb dieser Zusammenhänge auch wieder sein Recht.

Zum Schlusz: Reformierte Christen haben immer benachdruckt, dass die rechte Liturgie im weltlichen täglichen Leben stattfindet. Grundtvigs Nachdruck auf das "Mundwort“ des Herrn bei Taufe und Abendmahl scheint ihnen zu einer von der Welt abgewandten Kultgemeinschaft zu führen. Es ist wichtig, ganz deutlich zu machen, dass Grundtvigs christliches Denken ihn nicht vom irdischen konkret-menschlichen Leben isoliert, sondern ihn gerade zur innigsten Teilnahme führt. Der Herr, den wir mit Gebet, Bekenntnis und Lobgesang im Gottesdienst begegnen, ist der Erlöser nicht aús dem bunten Leben draussen, sondern Er erlöst gerade dieses von $\mathrm{Ihm}$ geschaffene Leben selbst. Hier gibt es für reformatorische Christen, die gewöhnt sind dem Luthertum eine scharfe Scheidung von Kirche und Welt vorzuwerfen, wichtige Anknüpfungspunkte und zugleich Ansätze zur Selbstkritik, gerade weil Grundtvig den calvinistischen Lösungen für das Problem des Verhältnisses Kirche - Welt gegenüber mit seinem „Menneske først og Christen så “ einen eigenen Weg geht.

Aber das Alles auszuarbeiten würde diesmal zu weit führen. Ich möchte nur sagen, dass auch hier ein wichtiger Unterschied zwischen reformiertem und lutherischem Denken vorliegt, und aufgeklärt werden muss, diesmal betreffs der Fragen nach dem Verhältnis zwischen Gesetz und Evangelium. Vielleicht gibt es später eine Gelegenheit, die zweite Hauptthese Grundtvigs unter reformiertem Gesichtswinkel näher zu betrachten! 